\title{
Brush Cytology- Alternative to Endoscopic Biopsy in Diagnosing Malignancy
}

\author{
Parveen Malhotra*, Ritesh Kumar, Sonia Chhabra, Vani Malhotra, Yogesh Sanwariya and Isha Pahuja \\ Department of Medical Gastroenterology, Pathology, Obstetrics and Gynaecology, India
}

Submission: February 24, 2020; Published: July 23, 2020

*Corresponding author: Parveen Malhotra, Department of Medical Gastroenterology, PGIMS, Rohtak-124001, Haryana, India

\begin{abstract}
Introduction: Upper gastrointestinal tract lesions are the leading cause of morbidity and mortality worldwide. Upper gastrointestinal symptoms include dysphagia, vomiting, anorexia, retrosternal pain, weight loss and mass abdomen and patients may present with visible red and white mucosal lesions, ulcers, polypoidal or ulcerative growth in upper gastrointestinal tract (GIT)

Observation: Fifty patients suspected of having upper gastrointestinal malignancies, from the gastroenterology ward/OPD were included in the study.

Results: In our study, maximum number of cases were in the age group of 41-60 years with the mean age being 58 years. Male to female ratio was approximately 2:1. Maximum number of patients (42 cases) presented with dysphagia

Conclusion: Although endoscopic biopsies are an established gold standard for diagnosing gastrointestinal malignancies but brush cytology can be used as an accurate diagnostic adjunct to biopsy in diagnosis of cancer and an important screening technique for detecting upper gastrointestinal malignancy in high risk population and in cases where difficulty is encountered in obtaining adequate tissue for histopathological examination.
\end{abstract}

Keywords: Cytology; Endoscopy; Brush biopsy

\section{Introduction}

Upper gastrointestinal tract lesions are the leading cause of morbidity and mortality worldwide. Upper gastrointestinal symptoms include dysphagia, vomiting, anorexia, retrosternal pain, weight loss and mass abdomen and patients may present with visible red and white mucosal lesions, ulcers, polypoidal or ulcerative growth in upper gastrointestinal tract (GIT) [1]. The pattern of primary cancers differ in different regions of the world depending upon the genetic, cultural, dietary and socioeconomic factor [2]. Upper gastrointestinal tract is a common site for malignant tumours among which gastric adenocarcinoma is most common $[3,4]$. Worldwide gastric adenocarcinoma is the second most common cancer and carcinoma esophagus is sixth leading cause of death [5]. The prevalence of oesophageal cancer has increased six times in the recent three decades, which is the most rapid increase amongst major malignancies [6]. In India, according to National Cancer Registery, oesophageal and gastric cancers are the most common cancers found in men while oesophageal cancer ranks third among women after carcinoma of breast and cervix [7]. Colo-rectal cancer is the third most common malignant diseases and the second most common frequent cause of cancer death in the United States [8]. Worldwide colorectal cancer is the third most commonly diagnosed malignant disease [9]. An early detection of oesophageal cancers gives five year survival rate of $83.5 \%$ and that of gastric cancer gives more than $90 \%$ five year survival rate [10].

The advent of endoscopy and endoscopic biopsy has greatly facilitated the detection and diagnosis of gastrointestinal neoplasms [5]. GIT lesions can be detected in many ways such as endoscopic guided balloon abrasive technique, lavage, or fine needle aspiration performed under either endoscopic guidance, or computed tomographic guidance [11]. However, the diagnostic value of cytology has been less recognized in the evaluation of these lesions. The use of cytology in addition to biopsy still remains controversial, as it appears to duplicate biopsy [5].

In 1964, Kobayashi $\mathrm{S}$ et al introduced brushing cytology under direct vision using fiberoptic gastroscope [12]. Brush cytology often complements and increases the sensitivity and specificity of detection of GIT lesions in many ways6. Brushing can yield near-tissue equivalent diagnostic material that can form the basis for therapeutic decisions. Minimal morbidity, low cost, 
and a rapid turn around time that reduces patient anxiety make adjuvant brush cytology along with biopsy, a preferred method in the evaluation of gastrointestinal malignancy [13]. It is useful in narrow, strictured lesions where access to the tumor by the biopsy forceps is limited and broader surface area can be accessed as compared to biopsy [14].

\section{Review of Literature}

The highest incidence of malignancy is in the esophagus, stomach and colorectal regions. In fact, esophagogastric and colorectal malignancies are amongst the commonest cancers in humans [15]. Over the last years the use of gastrointestinal cytology has declined due to preference for tissue biopsies [16] however, cytologic evaluation is often complementary to histologic diagnosis and is quite accurate and safe in expert hands [17]. Cytological smears show numerous small clusters and glandular groups with overlapping and loss of polarity. Loosely cohesive cells and scattered single cells may be seen in a necrotic background. The cytoplasm is variable in amount, delicate, finely granular and may show vacuolation. The tumor cell nuclei are enlarged, pleomorphic, have irregular nuclear membranes and show prominent nucleoli [15].

Vidyavathi $\mathrm{K}$ et al, [5] did a study to find correlation of endoscopic brush cytology with endoscopic biopsy in diagnosis of upper gastrointestinal neoplasms. Seventy five patients with upper gastrointestinal symptoms such as dysphagia, vomiting, retrosternal pain, anorexia, loss of weight and mass abdomen were subjected to endoscopy over a period of 2 years. Patients with visible mucosal lesions such as ulcer, polypoid or ulcerative growth in the upper GIT were included in the study. After visual examination of the lesion, cytology is performed using a small brush which is introduced through a separate channel in the endoscope. Six smears were made by directly smearing the brush onto a slide. Four slides were fixed with a spray fixative containing 95\% ethyl alcohol in carbowax and were stained by haematoxylin and eosin and Papanicolaou stain. Two slides were air-dried and stained with May-Grunwald-Giemsa stain. After brushing, multiple biopsies were taken from the surface and margins of the suspicious lesion. The tissue fragments were fixed in $10 \%$ buffered formalin and processed routinely. Histological sections were routinely stained by haematoxylin and eosin method. Histopathological and cytological findings were compared. Out of 75 cases, brush cytology was positive for malignancy in 65 cases $(86.66 \%)$ and biopsy was positive in 58 cases $(77.33 \%)$; thereby suggesting brush cytology as a useful adjunct to biopsy in the diagnosis of upper gastrointestinal tract malignancy. The study concluded that though biopsy is used as a routine procedure in diagnosis of gastrointestinal tract lesions, cytology is useful because it is inexpensive, gives a rapid diagnosis and offers minimal discomfort to the patient [5].

\section{Aim and Objectives}

a) To evaluate the utility of endoscopic brush cytology in diagnosing upper gastrointestinal malignancies.

b) To compare endoscopic brush cytology with endoscopy biopsy.

c) To assess the utility of endoscopic brush cytology as an adjunct to endoscopic biopsy in diagnosis of upper gastrointestinal malignancies.

\section{Material and Methods}

The present prospective study was conducted in the Department of Pathology in collaboration with Department of Gastroenterology at Pt. B.D. Sharma PGIMS, Rohtak. A total of fifty patients suspected of having upper gastrointestinal malignancies formed the study group. After taking the detailed history, patients were subjected to endoscopy using flexible video endoscope. After visual examination of the lesion, a cytological brush made up of small nylon bristles at the tip was introduced through a separate channel in the endoscope. The brush was advanced up to the lesions so that the bristles touched the mucosa or suspected lesion, by up and down or rotary motions. The trapped exfoliated cells were smeared on minimum of two glass slides. Half of the slides were air dried and others were fixed in 95\% alcohol. Air dried slides were stained with Romanowsky stain and alcohol fixed slides were stained with Haematoxylin and Eosin/Papanicolaou stain. After brushing, biopsies were taken from the lesions and preserved in $10 \%$ formalin. Biopsies were processed by routine histopathological technique and paraffin sections obtained were stained with Haematoxylin and Eosin (H\&E) stains. Smears and sections were interpreted as negative, suspicious of malignancy and positive for malignancy.

\section{Analytical Statistics}

The data was subsequently analysed using SPSS software (Version 21.0). Chi-square test and other relevant statistics (including sensitivity, specificity, positive predictive value and negative predictive value) were used. P-value less than 0.05 was accepted as statistically significant.

\section{Observation}

Fifty patients suspected of having upper gastrointestinal malignancies, from the gastroenterology ward/OPD were included in the study (Figure 1-12).

Thus, brush cytology and histopathological findings on biopsy were compared and the accuracy of brush cytology for detection of malignant lesions was evaluated taking histopathology as the reference standard test. The results were interpreted in the form of sensitivity (likelihood that patient with the disease has positive test results), specificity (likelihood that patient without the disease has negative test results), positive predictive value (the probability that patients with a positive screening test truly have the disease), negative predictive value (the probability that patients with a negative screening test truly do not have the disease). 


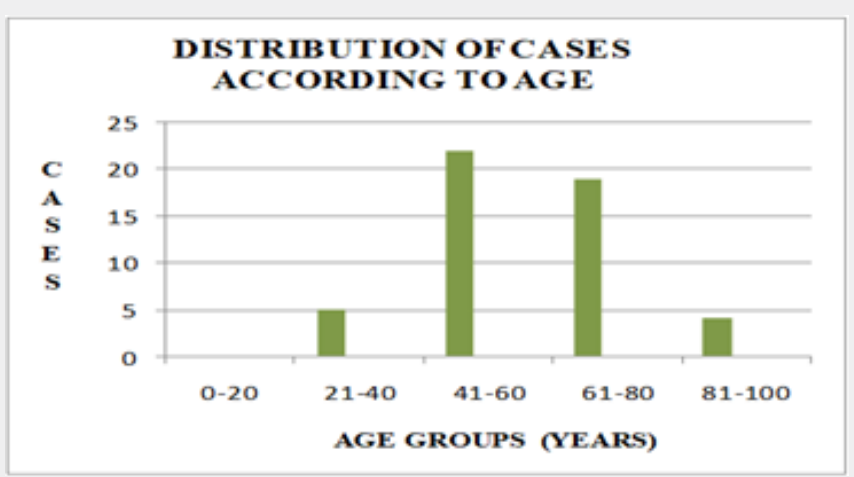

Figure 1: Distribution of cases according to age.

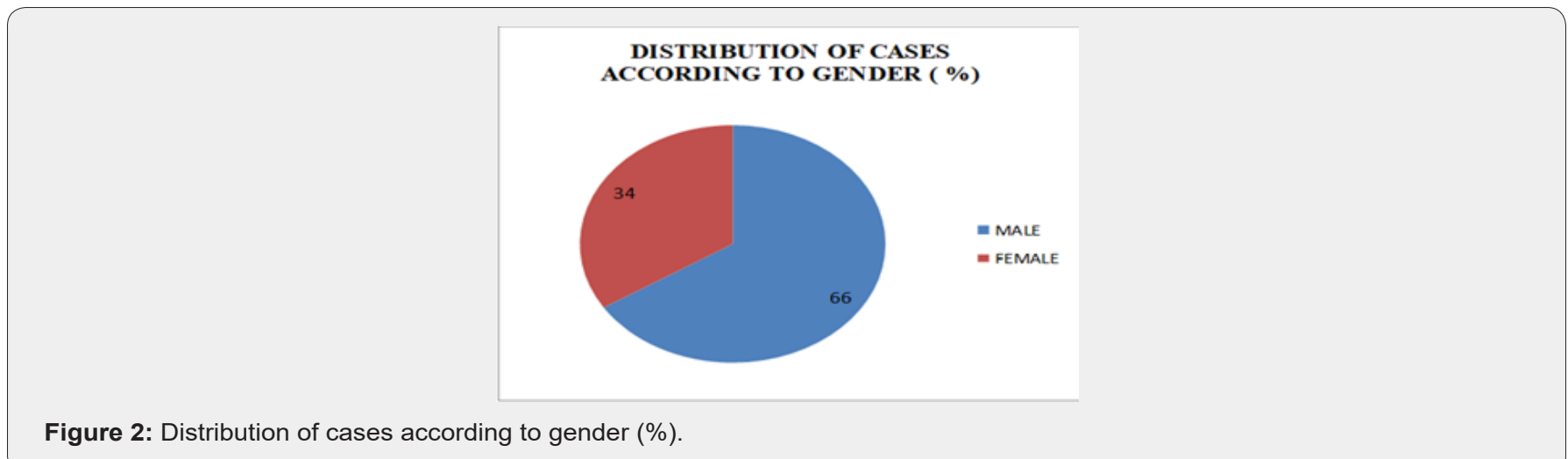

Figure 2: Distribution of cases according to gender (\%).



Figure 3: Distribution of cases according to clinical complaints.

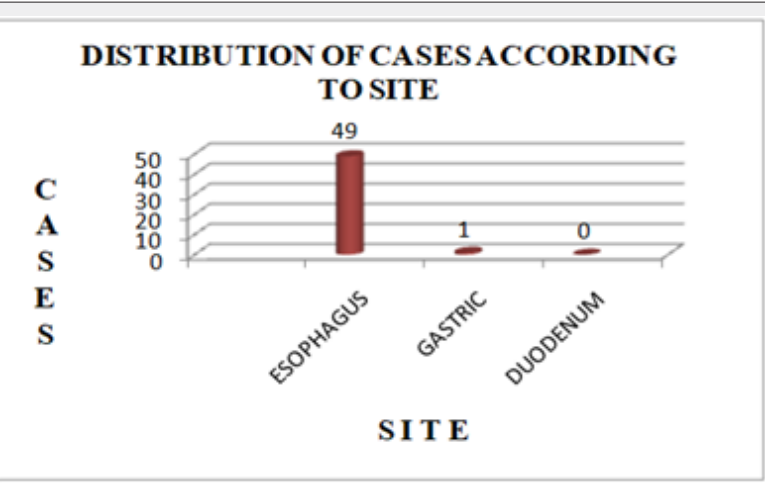

Figure 4: Current Trends in Biomedical Engineering \& Biosciences 


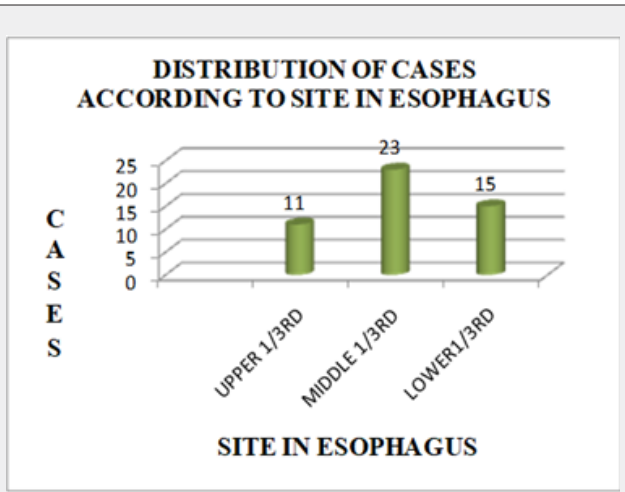

Figure 5: Distribution of cases according to site in esophagus.

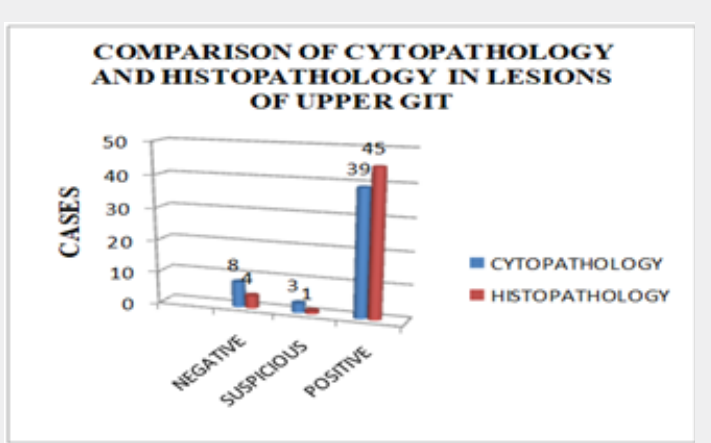

Figure 6: Current Trends in Biomedical Engineering \& Biosciences

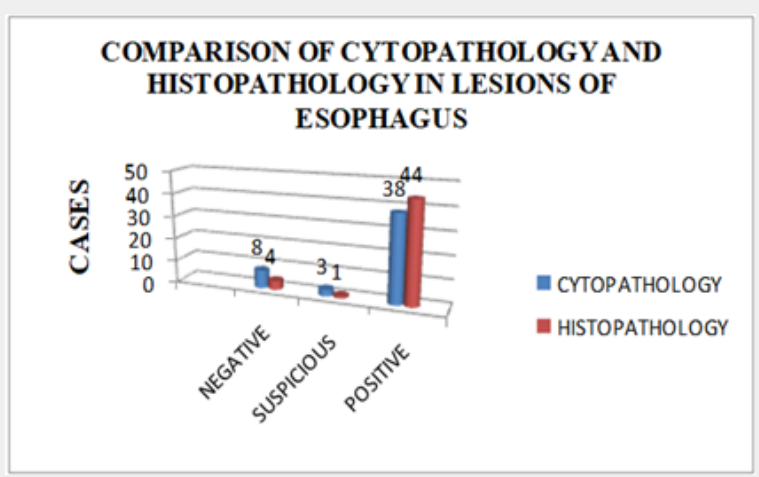

Figure 7: Comparison of cytopathology and histopathology in lesions of esophagus.

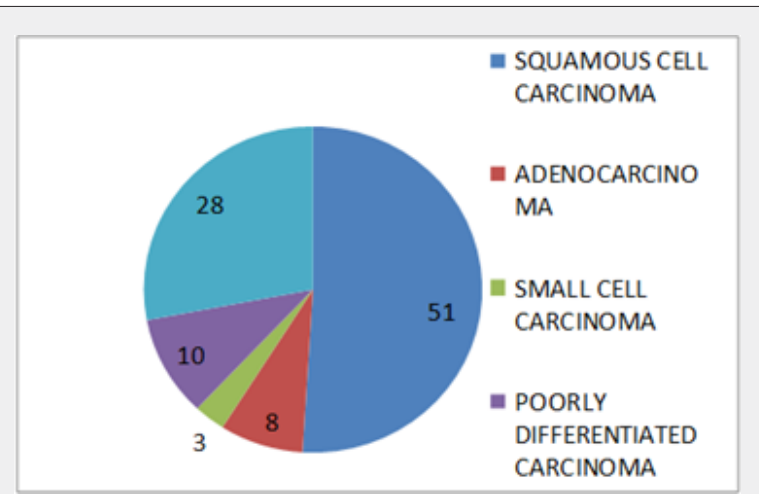

Figure 8: Types of carcinoma diagnosed on cytopathology. 


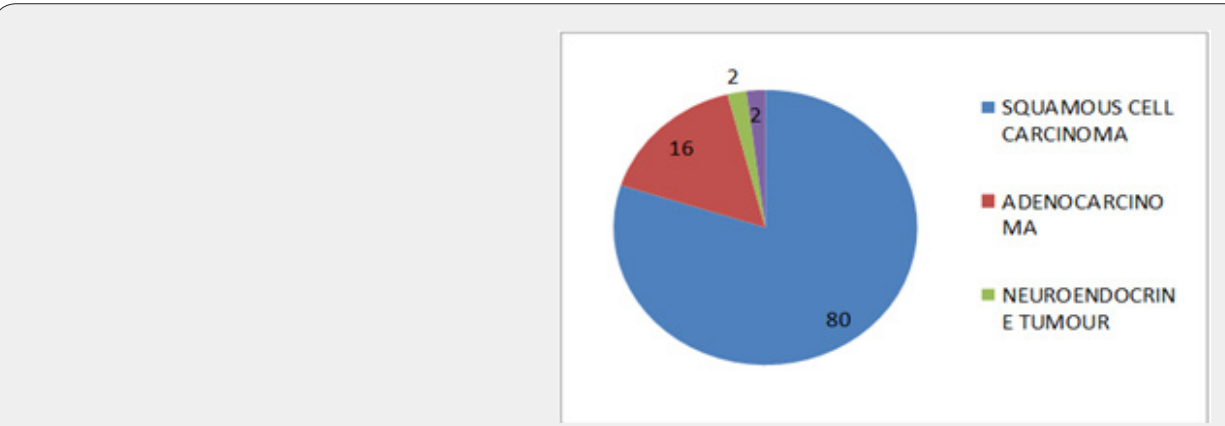

Figure 9: Types of carcinoma diagnosed on histopathology (\%).

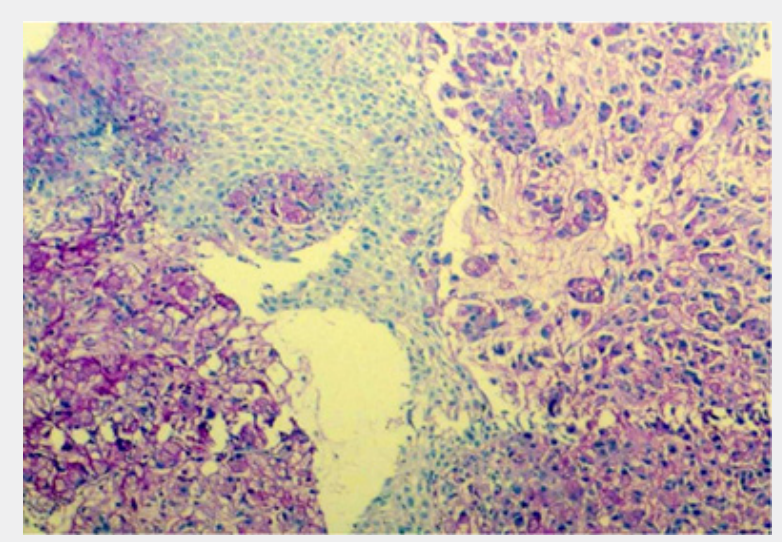

Figure 10: Mucin secreting adenocarcinoma (PAS, 100X).

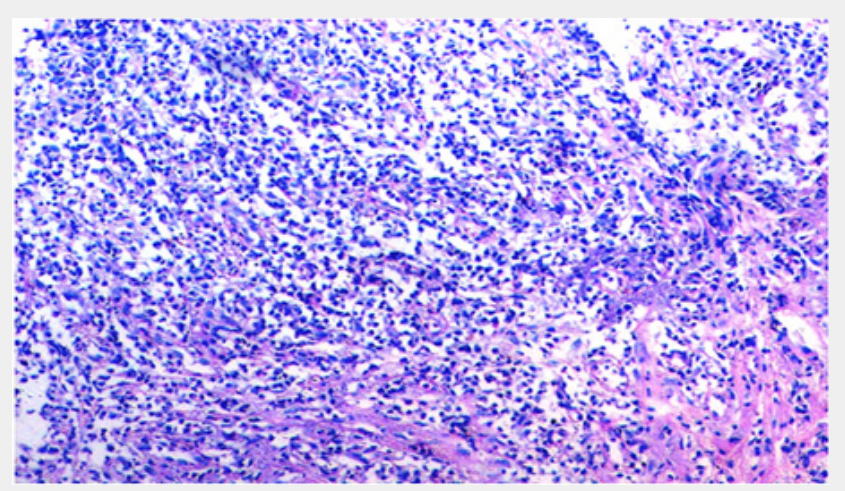

Figure 11: Poorly differentiated squamous cell carcinoma (H\&E, 100X).

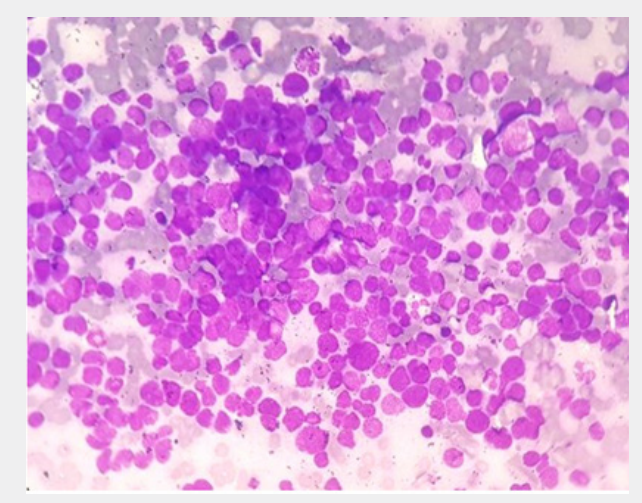

Figure 12: Small cell carcinoma esophagus (Romanowsky Stain, 200X). 
The sensitivity and positive predictive value in our study is $84.4 \%$ and $97.4 \%$ respectively while the specificity and negative predictive value is $100 \%$ and $50 \%$ respectively which means in an adequate brush cytology smear, malignancy was never over diagnosed. These findings substantiate the value of brush cytology in diagnosing upper gastrointestinal malignancies.

\section{Discussion}

Gastrointestinal malignancies are a leading cause of morbidity and mortality worldwide. In India, according to National cancer registery, oesophageal and gastric cancers are the most common cancers found in men, while oesophageal cancer ranks third among women after the carcinoma of breast and cervix [7]. Early lesions are asymptomatic and highly curable, but unfortunately most of them are diagnosed in advanced stages of disease. The introduction of flexible fibre optic endoscope has greatly expanded the usefulness and precision of gastrointestinal tract cytology [5]. So direct vision brush cytology of upper GIT mucosa with flexible endoscope is now a standard method of diagnosis independently or as an adjunct to tissue biopsy. Its reliability has been reported to range from $75 \%$ to $90 \%$ in various studies [18]. The primary role of gastrointestinal cytology is in cancer detection. Endoscopy allows the visualization of mucosal lesions and at same time permits the sampling of cytology and biopsy for a definitive diagnosis. It is an outpatient procedure and is considered minimally invasive and does not require any significant recovery after the procedure [1].

During our study period, 50 cases suspected of upper gastrointestinal malignancies were included in which brush cytology followed by biopsy were performed. Patients were divided into various age groups ranging from 0-20, 21-40, 41-60, 61-80 and 81-100 years. In our study, majority of patients were in the age group of 41-60 years with the mean age being 58 years. The mean age is comparable to studies conducted by Vidyavathi \& Kaur et al. [5,11].

In our study, the total male count diagnosed with upper gastrointestinal cancer was 33 and the female count was 17 with male to female ratio almost 2:1. This ratio is in conformity with findings of Vidyavathi \& Kaur et al. $[5,11]$ who reported a male: female ratio of 2.5:1 and 1.7:1 respectively. In all the studies, males were found to be affected more commonly as compared to females. The reason for this finding is that males are comparatively more exposed to environmental pollutants as compared to females and are more indulged in habits of smoking and tobacco consumption as compared to females.

In our study, dysphagia was most common clinical complaint found in 42 patients (84\%) followed by dysphagia with epigastric pain in 4 patients (8\%) and dysphagia with vomiting in 4 cases (8\%). This is in accordance with the study of Karmarkar et al. [1] who also reported dysphagia to be the most common clinical complaint in patients of upper gastrointestinal malignancies. Vidyavathi et al, [5] however reported abdominal pain to be the most common symptom (38.66\%) followed by dysphagia in
$34.61 \%$ patients because maximum cases in his study were of gastric lesions (64\%) followed by oesophageal lesions (30.66\%).

Esophagus was the most common site of involvement accounting for 49 out of 50 cases in this study. One case was reported to be of gastric malignancy. This is in accordance with Karmarkar et al. [1] whose study showed $94 \%$ cases of oesophageal lesions, 5\% gastric lesions and 1\% duodenal lesion. Vidyavathi et al, [5] however reported predominance of gastric lesions in her study (64\%) followed by oesophageal (30.66\%) and duodenal (5.33\%) lesions. This difference is attributed to various studies being carried in different geographical areas with wide difference in habits of the people living in these areas.

The lesion was located in upper $1 / 3^{\text {rd }}$ of the esophagus in 11 patients, middle $1 / 3^{\text {rd }}$ in 23 patients and lower $1 / 3^{\text {rd }}$ in 15 patients. This finding was in accordance with the Vidyavathi et al, [5] whose study also showed middle $1 / 3^{\text {rd }}$ as the predominant site Kaur et al, [11].

In our study, out of 45 cases positive for malignancy on biopsy in upper GIT, 36 (80\%) cases were squamous cell carcinoma (SCC), $7(16 \%)$ cases were adenocarcinoma, 1 was of neuroendocrine tumour $(2 \%)$ and 1 case was of poorly differentiated carcinoma (2\%). Out of 39 cases positive for malignancy on cytology [20] (51\%) cases were squamous cell carcinoma, 3 (8\%) were adenocarcinoma, $1(3 \%)$ was of small cell carcinoma, 4 (10\%) cases were poorly differentiated carcinoma and 11(28\%) were unspecified carcinoma. Studies by Kaur \& Vidyavathi et al, [5,11] also showed squamous cell carcinoma being the most common malignancy in upper GIT.

Statistical analysis revealed the overall sensitivity of brush cytology in this study to be $84.4 \%$ which emphasizes the usefulness of brush cytology as a screening procedure. The sensitivity of this study is comparable to studies of Kaur et al, [11] having the diagnostic sensitivity of $80.95 \%$ \& Vidyavathi et al, [5] having $98 \%$. Many factors influence the diagnostic accuracy of brush cytology. In order to improve the positive rate of brush cytology examination, some technical considerations should be noted such as appropriate selection of the site for the specimen, avoiding areas covered by thick slough or necrotic material and the procedure should be performed by a technically trained person. It should be performed at the margin of the lesion and the entire head of nylon brush should be used over a wide area with appropriate force. After brushing is completed, the head of brush should be withdrawn to the tip to prevent the loss of specimen. During smearing of the slides, filter paper should be used to absorb excess mucus or clot adherent to the head of the brush [5]. Considering these points, endoscopic brush cytology can emerge as an effective method for evaluating and screening upper gastrointestinal lesions and can be used as an alternative to biopsy, that is an invasive technique, for rapid diagnosis with minimal discomfort to patients though it is not able to differentiate characteristically between dysplasia, in situ and invasive malignancy. 


\section{Summary and Conclusion}

In our study, maximum number of cases were in the age group of 41-60 years with the mean age being 58 years. Male to female ratio was approximately 2:1. Maximum number of patients (42 cases) presented with dysphagia. Dysphagia with epigastric pain was seen in 4 cases and the rest presented with dysphagia with vomiting. Esophagus was the most common site of involvement accounting for 49 out of 50 cases and a single case was of gastric lesion. Maximum number of oesophageal malignancies (23 cases) were located in middle $1 / 3^{\text {rd }}$ followed by lower $1 / 3^{\text {rd }}$ in 15 cases and upper $1 / 3^{\text {rd }}$ in 11 cases. Out of 39 cases positive for malignancy on brush cytology, $51 \%$ were squamous cell carcinoma, $8 \%$ were adenocarcinoma, 3\% were small cell carcinoma, $10 \%$ were poorly differentiated carcinoma and $28 \%$ cases were diagnosed as carcinoma (unspecified). Out of 45 cases positive for malignancy on biopsy, 80\% were squamous cell carcinoma, $16 \%$ were adenocarcinoma, $2 \%$ neuroendocrine tumor and $2 \%$ poorly differentiated carcinoma. The overall sensitivity of brush cytology in our study was $84.4 \%$ which emphasizes the usefulness of brush cytology as a screening procedure. The overall specificity of brush cytology in our study was $100 \%$, meaning by that the patients without disease have negative test results. The positive predictive value of cytology in our study was $97.4 \%$ which signifies that in an adequate brush cytology smear, malignancy was never over diagnosed. The negative predictive value in our study was $50 \%$. To conclude, brush cytology is a reliable, simple, safe, rapid, non-invasive yet effective, inexpensive method of detecting malignancy of upper gastrointestinal tract. A large surface area can be sampled and the technique has high specificity and good sensitivity. With increased experience, meticulous care in technique and adherence to strict criteria for malignancy and by identifying a suspicious category, malignancy can be effectively detected and treated and it can drastically alter the outcome in terms of morbidity and mortality. Although endoscopic biopsies are an established gold standard for diagnosing gastrointestinal malignancies but brush cytology can be used as an accurate diagnostic adjunct to biopsy in diagnosis of cancer and an important screening technique for detecting upper gastrointestinal malignancy in high risk population and in cases where difficulty is encountered in obtaining adequate tissue for histopathological examination.

\section{Future Implications}

Brush cytology can be used as an independent technique for screening and early detection of oesophageal malignancy among high risk population and in high risk patients with known premalignant conditions like Barrett's esophagus, cardiac achalasia etc. and for clinical follow up. With increased expertise in procedure and reporting, it can emerge as an effective diagnostic modality in mass screening projects..

\section{References}

1. Karmarkar P, Wilkinson A, Manohar T, Joshi A, Mahore S (2013) Diagnostic utility of endoscopic brush cytology in upper gastrointestinal lesions and its correlation with biopsy. IOSR J Dental Med Sci 5(2): 32-36.

2. Al Diab J, Sahib FA, Strak SK (2005) Endoscopic biopsy versus brush cytology in the diagnosis of various gastrointestinal diseases with special reference to gastrointestinal tumors. Bas J Surg 11(2): 15-18.

3. Zhang XF, Huang CM, Lu HS, Wu XY, Wang C, et al. (2004) Surgical treatment and prognosis of gastric cancer in 2613 patients. World J Gastroenterol 10(23): 3405-3408.

4. Enzinger PC, Mayer RJ (2003) Oesophageal cancer. N Engl J Med 349(23): 2241-2252.

5. Vidyavathi K, Harendrakumar ML, Lakshmana kumar YC (2008) Correlation of endoscopic brush cytology with biopsy in diagnosis of upper gastrointestinal lesions. Indian J Pathol Microbiol 51(4): 489492.

6. Flejou JF (2005) Berrett's esophagus: from metaplasia to dysplasia and cancer. Gut 54: 6-12.

7. Salpekar K, Mahore S, Karmarkar P (2014) Endoscopic brush cytology: a screening and surveillance tool for gastrointestinal malignancy. IOSR J Dental Med Sci 58-63.

8. Jemal A, Murray T, Ward E (2005) Cancer Statistics, 2005. CA Cancer J Clin 55: 10-30.

9. Parkin DM, Bray F, Ferlay J, Pisani P (2005) Globocan 2002: Cancer incidence, mortality and prevalence worldwide. CA Cancer J Clin 55: 74-108.

10. Kato H, Tachimori Y, Watanube H, Yamaguchi H, Ishikawa J, et al. (1990) Superficial esophageal carcinoma: Surgical Treatment and results. Cancer 66: 2319-2323.

11. Kaur S, Sharma R, Kaushal V, Gulati A, Sharma B (2016) Diagnostic accuracy of endoscopic brush cytology in malignancies of upper gastrointestinal tract: a prospective study of 251 patients in North India. J Cancer Res Ther 12(2): 681-684.

12. Kobayashi S, Kasugai T (1978) Brushing cytology for the diagnosis of gastric cancer involving the cardia or the lower esophagus. Acta Cytol 22(3): 155-157.

13. Xing GS, Geng JC, Han XW, Dai JH, Wu CY (2005) Endobiliary brush cytology during percutaneous transhepatic cholangiodrainage in patients with obstructive jaundice. Hepatobiliary Pancreat Dis Int 4(1): 98-103.

14. Kaminsky DB (1984) Aspiration biopsy in the context of the new Medicare fiscal policy. Acta Cytol 28(3): 333-336.

15. Conrad R, Prabhu SC, Cobb C, Razza A (2012) Role of cytopathology in the diagnosis and management of gastrointestinal tract cancers. J Gastrointest Oncol 3(3): 285-298.

16. (1973) The Coordinating Group for the Research of Esophageal carcinoma. Chinese Academy of Medical Sciences and Honan Province. The early detection of carcinoma of the esophagus. Scientia Sinica 16: 457-463.

17. Morenootero R, Martinezraposo A, Cantero J, Pajares JM (1988) Exfoliative cytodiagnosis of gastric adenocarcinoma comparison with biopsy and endoscopy. Acta Cytol 27: 458-488.

18. Dowlatshahi K, Skinner DB, Demeester TR, Zachary L, Bibbo M, et al. (1985) Evaluation of brush cytology as an independant technique for detection of esophageal carcinoma. J Thorac Cardiovasc Surg 89(6): 848-851. 
